\title{
An Experimental Study of Muscle Coordination and Function during Human Locomotion
}

\author{
Keita Inoue Taiki Iimura Takanori Oku Hang T.T. Pham Hiroaki Hirai Fumio Miyazaki \\ Graduate School of Engineering Science, Osaka University, Japan \\ E-mail: \{inoue, iimura, oku, phamhangtt, hirai, miyazaki\}@robotics.me.es.osaka-u.ac.jp
}

\begin{abstract}
How humans solve the ill-posed problem of motor control is still a mystery. In this paper, we attempt to decompose human walking and running as the main movements of a leg into units of motor function. We introduce the key concept of "A-A ratio," defined as the ratio of an extensor muscle's electromyography (EMG) signal to the sum of agonist and antagonist muscles' EMG signals. Human walking and running are then decomposed into two units of motor function by applying Principal Component Analysis (PCA) to the A-A ratio dataset. The kinematic meanings of these units are also experimentally shown by using a human-like musculoskeletal leg robot.
\end{abstract}

\section{Introduction}

In neuroscience, an enormous amount of research has focused on decomposing motor commands into some functional units or synergies, which are important for solving Bernstein's problem of redundant degrees of freedom (DFs) [1], [2]. It regards the central nervous system's (CNS's) commands to muscle groups as units of motor function to achieve movement. However, these studies discussed the less physical meaning of these synergies. Meanwhile, in an effort to explore the solution to Bernstein's problem, we also decomposed human walking and running by using our original method, and revealed the physical meaning of units of motor function [3], [4]. In our previous study, we introduced the concept of "the agonist-antagonist muscle pairs (A-A) ratio," defined as the ratio of EMG signals for the agonist and antagonist muscles, and decomposed human walking into two kinematic motor functions by applying Principal Component Analysis (PCA) to the A-A ratio data set [3]. We also succeeded in decomposing human running into two motor functions using the similar method [4]. These two results, however, could not be compared directly because they were obtained by slightly different analysis methods of the A-A ratio.

In this paper, we analyze human walking and running using the same method. We propose the normalized A-A ratio, which represents the coordination of agonist-antagonist muscle pairs. The normalized A-A ratio is defined as the ratio of an extensor muscle's EMG signal to the sum of agonist and antagonist muscles' EMG signals. The normalized A-A ratio based on the EMG signals during human walking and running is decomposed into two principal components (PCs) by using the PCA technique. We especially analyze the $\mathrm{PC}$ vectors of the A-A ratio, and demonstrate that (1) human walking and running can be decomposed into two patterns of muscle coordination associated with kinematic motion; (2) the extracted patterns do not depend on whether the movement is walking or running. The physical functions of the muscle coordination are also experimentally clarified by transferring the extracted muscle patterns into a human-like musculoskeletal robot.

\section{Materials and methods}

\subsection{Motion measurement}

Two healthy subjects, A (male, 23 years old, $1.75 \mathrm{~m}$, $60.4 \mathrm{~kg}$ ) and B (male, 23 years old, $1.74 \mathrm{~m}, 60.0 \mathrm{~kg}$ ), volunteered for the experiment. Each subject walked on a treadmill (SportsArt Fitness T650m) at natural speed (subject A, $3.5 \mathrm{~km} / \mathrm{h} ; \mathrm{B}, 3.9 \mathrm{~km} / \mathrm{h}$ ) and $5.0 \mathrm{~km} / \mathrm{h}$, and ran on it at 7.0 and $9.0 \mathrm{~km} / \mathrm{h}$ for $30 \mathrm{sec}$.

The surface EMG activities of eight muscles of the left leg were recorded using a multi-telemeter system, WEB-5000 (Nihon Koden), at $1000 \mathrm{~Hz}$. This system sends EMG data to a computer after band-pass filtering $(0.03$ to $450 \mathrm{~Hz})$, anti-hum filtering (around $60 \mathrm{~Hz})$, and amplification $(\times 2000)$. The eight analyzed muscles are shown in Fig. 1. These mono-/bi- articular muscles are known as the major muscles relevant to hip, knee, and

This is an Open Access article distributed under the terms of the Creative Commons Attribution-Noncommercial License 3.0, which permits unrestricted use, distribution, and reproduction in any noncommercial medium, provided the original work is properly cited. 


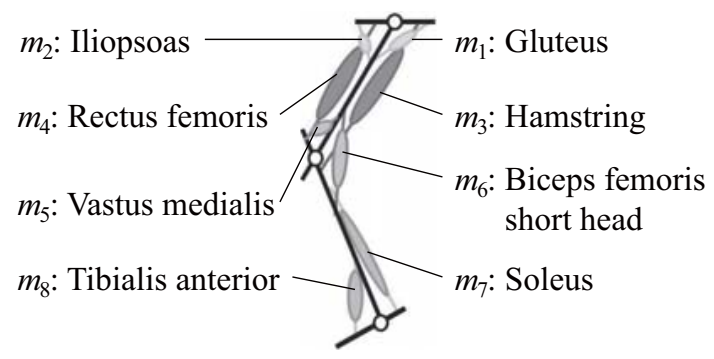

Fig. 1. Musculoskeletal model of human lower limb

ankle joint movements. The skin areas were cleaned with alcohol and abraded in order to reduce skin resistance $(<10 \mathrm{k} \Omega)$. The interelectrode distance was $2 \mathrm{~cm}$.

A gait and run cycle was defined with respect to the left leg movement, beginning with left-heel contact with the ground (gait phase $0 \%$ ) and ending with next leftheel contact (gait phase 100\%).

\subsection{Data analysis}

Data analysis was performed after the following preparations for raw EMG data obtained by motion measurement: band-pass filtering (20 to $450 \mathrm{~Hz}$ ), fullwave rectification, smoothing, and normalization to Maximum Voluntary Contraction (\%MVC). The EMG signal of muscle for the experiment is expressed as $m_{i}$ $(i=1, \cdots, 8)$ (Fig. 1). The \%MVC data was averaged by using 12 to 30 gait and run cycles.

In this study, we focus on the coordination between the agonist and antagonist muscles to define the A-A ratio $r_{i}(i=1, \cdots, 4)$ (Table 1$)$. This definition enables analysis of parameters that contribute to the joint angle movement. For example, when a hip joint is extending, the extensor muscle's EMG $\left(m_{1}\right)$ increases and the flexor muscle's EMG $\left(m_{2}\right)$ decreases, resulting in an increase in the A-A ratio $r_{1}$. For this reason, the A-A ratio $r_{1}$ can be said to contribute to the hip joint angle movement. From here on, we analyze human walking and running by using this parameter based on EMG signals.

The A-A ratio data set for the gait or run cycle is expressed as $\boldsymbol{D}=\left\{r_{j}\left(t_{i}\right)\right\}$ (a $p \times q$ matrix, where $r_{j}\left(t_{i}\right)$ is the $j$-th A-A ratio at time $t_{i}, p$ is the number of time points during a gait or run cycle, and $q$ is the number of labels of A-A ratios). In our experiment, $p=730$ to 1220 and $q=4$. The number $p$ varies according to the gait or run speed because of the varying gait or run cycle. Matrix $\boldsymbol{D}$ is then expressed as

$$
\boldsymbol{D}=\left[\begin{array}{cccc}
r_{1}\left(t_{1}\right) & r_{2}\left(t_{1}\right) & r_{3}\left(t_{1}\right) & r_{4}\left(t_{1}\right) \\
r_{1}\left(t_{2}\right) & r_{2}\left(t_{2}\right) & r_{3}\left(t_{2}\right) & r_{4}\left(t_{2}\right) \\
\vdots & \vdots & \vdots & \vdots \\
r_{1}\left(t_{p}\right) & r_{2}\left(t_{p}\right) & r_{3}\left(t_{p}\right) & r_{4}\left(t_{p}\right)
\end{array}\right]
$$

Table 1. Definition of the A-A Ratio

\begin{tabular}{|c|c|c|}
\hline Label & Target muscles & Movement function \\
\hline \hline$r_{1}$ & $m_{1} /\left(m_{1}+m_{2}\right)$ & Hip extension \\
\hline$r_{2}$ & $m_{3} /\left(m_{3}+m_{4}\right)$ & Hip extension and knee flexion \\
\hline$r_{3}$ & $m_{5} /\left(m_{5}+m_{6}\right)$ & Knee extension \\
\hline$r_{4}$ & $m_{7} /\left(m_{7}+m_{8}\right)$ & Ankle extension \\
\hline
\end{tabular}

Applying PCA to the A-A ratio data set $D$ results in the linear combination:

$$
\operatorname{diag}[\boldsymbol{\sigma}]^{-1}(\boldsymbol{r}(t)-\overline{\boldsymbol{r}})=\sum_{i=1}^{4} w_{i}(t) \boldsymbol{s}_{i}
$$

where $\boldsymbol{r}(t)=\left[r_{1}(t), r_{2}(t), r_{3}(t), r_{4}(t)\right]^{\mathrm{T}}$ is an A-A ratio data set vector, $\operatorname{diag}[\sigma]$ is a $4 \times 4$ diagonal matrix with $r_{i}$ 's standard deviation $\sigma_{i}$ in the $i$-th row and $i$-th column, $\bar{r}$ is the mean vector, $w_{i}(t)$ is the $i$-th PC score, and $\boldsymbol{s}_{i}=\left[s_{i 1}, s_{i 2}, s_{i 3}, s_{i 4}\right]^{\mathrm{T}}$ is the $i$-th 4-dimensional $\mathrm{PC}$ vector of the A-A ratio. The PC vectors of the A-A ratio $s_{i}$ present the units of motor function that describe the balance among the eight muscles.

\subsection{Transferring muscle coordination to robot}

To explore the intrinsic physical meaning of muscle coordination, we transferred the extracted PC vector patterns to a human-like musculoskeletal leg robot (Fig. 3). Our robot consists of a skeletal model (Avice, Inc.) and eight pneumatic artificial muscles (PAMs) (Kanda Tsushin Kogyo Co., Ltd.) corresponding to the examined muscles (Fig. 1). The robot's body parameters (e.g., segment mass and moment of inertia) are reconstructed from human body parameters [5] by attaching metal sheets to the robot. The muscle-attaching locations were decided by referring to human muscles. The robot has three degrees of freedom, with rotation of the hip, knee, and ankle joints in the sagittal plane. Air pressure supplied to the PAM is controlled by voltage commands from the computer to an air-pressure-control device (Hitachi Medical Corp.) that powers the pressure according to voltage changes via a proportional electromagnetic valve. The air-pressure command was determined based on two parameters of the robot: the A-A ratio $R_{i}$ and the agonist-antagonist muscle pair activity (A-A activity) $A_{i}(i=1,2,3,4)$. These parameters are defined as

$$
\left\{\begin{array}{l}
R_{i}=M_{2 i-1} /\left(M_{2 i-1}+M_{2 i}\right) \\
A_{i}=M_{2 i-1}+M_{2 i}
\end{array}\right.
$$

where $M_{2 i-1}$ and $M_{2 i}$ are air-pressure commands to the agonist-antagonist muscle pairs. Solving Eq. (3) for parameters $M_{2 i-1}$ and $M_{2 i}$ yields the following:

$$
\left\{\begin{array}{l}
M_{2 i-1}=R_{i} A_{i} \\
M_{2 i}=\left(1-R_{i}\right) A_{i}
\end{array}\right.
$$


Table 2. Contribution rates [\%] of the first and second $\mathrm{PCs}$ of the A-A ratio

\begin{tabular}{|c|cc|cc|cc|cc|}
\hline \multirow{3}{*}{ Subject } & \multicolumn{4}{|c|}{ Walking } & \multicolumn{4}{c|}{ Running } \\
\cline { 2 - 9 } & Natural & \multicolumn{2}{|c|}{$5 \mathrm{~km} / \mathrm{h}$} & \multicolumn{2}{|c|}{$7 \mathrm{~km} / \mathrm{h}$} & \multicolumn{2}{|c|}{$9 \mathrm{~km} / \mathrm{h}$} \\
\cline { 2 - 9 } & PC1 & PC2 & PC1 & PC2 & PC1 & PC2 & PC1 & PC2 \\
\hline \hline A & 64.3 & 30.2 & 73.4 & 23.5 & 65.3 & 33.3 & 55.3 & 43.4 \\
\hline B & 61.0 & 32.6 & 58.1 & 34.1 & 58.4 & 41.0 & 46.8 & 52.5 \\
\hline
\end{tabular}

Because $R_{i} \in[0,1]$, the A-A activity $A_{i}$ is the maximal air-pressure command to the PAMs. In our experiments, the A-A activity $A_{i}$ of all PAMs was fixed at an experimentally determined constant $\left(A_{i}=500[\mathrm{kPa}]\right)$ to refer to the kinematic meaning of the $\mathrm{PC}$ vectors of the A-A ratios. To transfer human muscle coordination of the AA ratios $\left(s_{i}\right)$ into the robot, we introduce the A-A ratio data set vector of the robot $\boldsymbol{R}(t)$. This parameter corresponds to the human A-A ratios. It is determined by using the first and second $\mathrm{PC}$ vectors of the human $\mathrm{A}-\mathrm{A}$ ratio as follows, according to Eq. (2).

$$
\boldsymbol{R}(t)=\sum_{i=1}^{4} W_{i}(t) \operatorname{diag}[\boldsymbol{\sigma}] \boldsymbol{s}_{i}+\overline{\boldsymbol{r}}
$$

Here, $W_{i}(t)$ is the $i$-th PC score of robot, and $\boldsymbol{R}(t)$ consists of the A-A ratios $R_{i}$ of artificial muscle pairs. Because the parameters $R_{i}$ of the muscle pairs can be determined in Eq. (5), it is possible to decide the airpressure commands in Eq. (4). $W_{i}(t)$ varies the size of the patterns of muscle coordination, and it corresponds to the PC score $w_{i}(t)$ of the human A-A ratio.

\section{Results}

The PCA technique decomposes the A-A ratio data set into a linear combination of muscle coordination (Eq. (2)). Figure 2 shows the PCA results of the subjects' A-A ratio. The first and second blocks from the top present the first and second $\mathrm{PC}$ vectors of the A-A ratio; the third block depicts the mean vector of the $\mathrm{A}-\mathrm{A}$ ratio; and the bottom block shows the first and second PC scores of the A-A ratio. PC scores are illustrated as circle-like trajectories in a $w_{1}-w_{2}$ plane. In all cases of our experiment (all locomotion rates and all subjects), the cumulative contribution rates up to the second PC exceed $92 \%$. Table 2 presents the contribution rates of the two PCs of the A-A ratio. This result indicates that the extracted two PCs can describe most of the eight muscles' activities.

To investigate the intrinsic physical meaning of muscle coordination, we transferred the extracted PC vector patterns to a human-like musculoskeletal leg robot. To visualize how the $\mathrm{PC}$ score contributes to the $\mathrm{PC}$ vector,

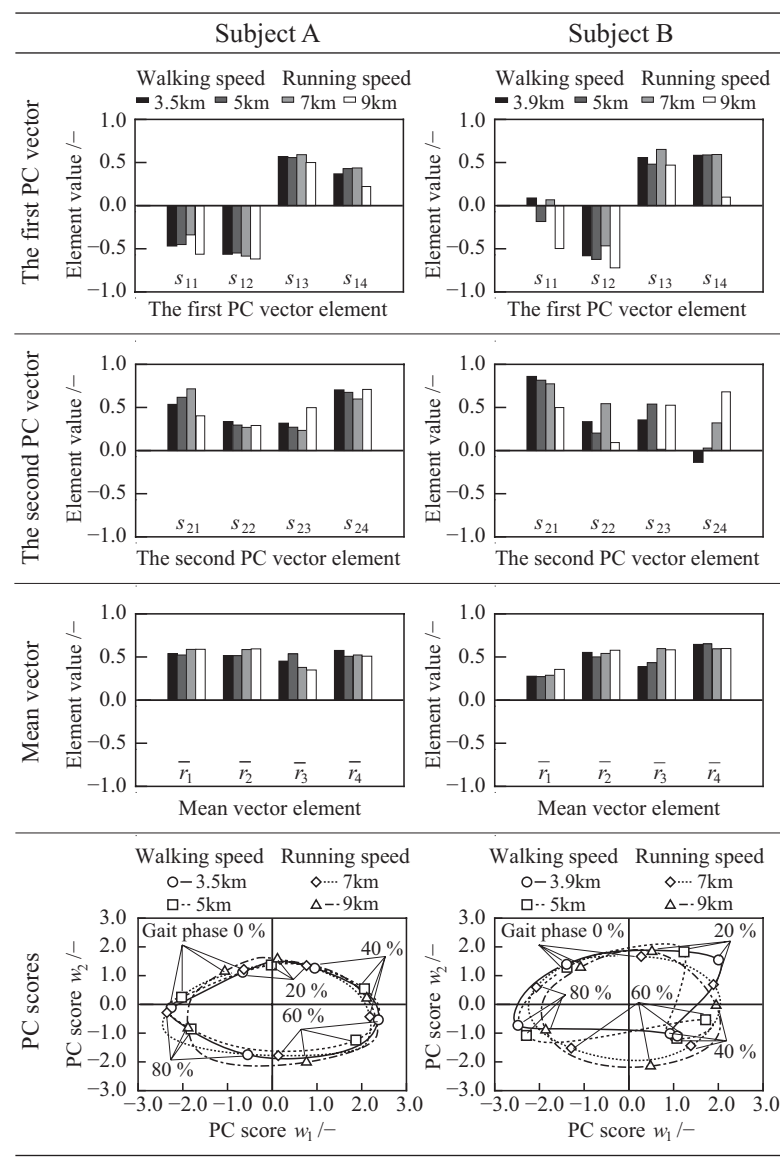

Fig. 2. PCA results of the $A-A$ ratio

we changed $W_{1}(t)$ or $W_{2}(t)$ linearly from -2.5 to 2.5 in 50sec so as to cover the range of $w_{1}(t)$ or $w_{2}(t)$ (see the bottom block in Fig. 2). Figure 3 illustrates the leg robot's movements generated by only the first or second $\mathrm{PC}$ vector pattern of muscle coordination for subject A's walking at $3.5 \mathrm{~km} / \mathrm{h}$ (natural speed).

\section{Discussion}

In this study, we applied PCA to the A-A ratio dataset based on the EMG signals during human walking and running, and decomposed these locomotions into two units of motor function presented by $\mathrm{PC}$ vectors of the A-A ratio. The inner product values of the first or second PC vectors through all the experiments (all locomotion rates) are close to 1.0 for each subject. For subject $\mathrm{A}$, the inner product values of the first or second PC vectors are $0.98 \pm 0.02$ and $0.97 \pm 0.03$, respectively; and for subject $\mathrm{B}$, those are $0.85 \pm 0.13$ and $0.75 \pm 0.12$, respectively. Moreover, the inner product values of each PC vector of both subjects through all 


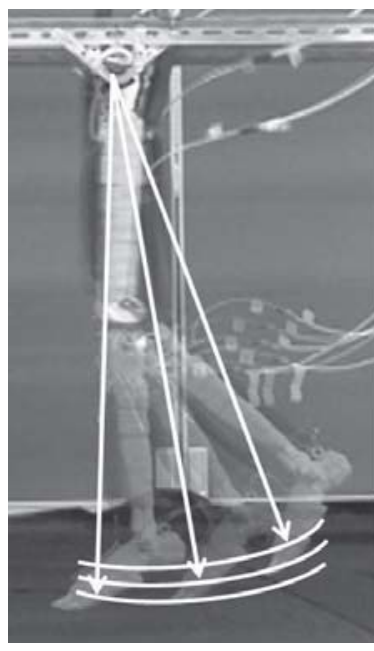

The first PC vector

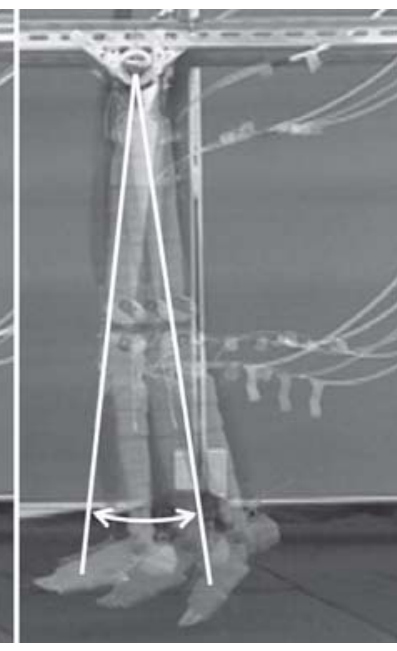

The second PC vector
Fig. 3. Physical meanings of muscle coordination

the experiments are also close to $1.0(0.91 \pm 0.06$ for the first PC vector and $0.80 \pm 0.15$ for the second PC vector). These facts indicate that each PC vector tends to be similar for different walking or running speeds and different subjects. This result implies that the bases of muscle coordination as units of motor function do not depend on locomotion speed, or on whether the subject is walking or running.

Furthermore, we transfered the extracted muscle patterns into a human-like musculoskeletal robot to explore the intrinsic physical meaning of muscle coordination. The proportional changes in the PC vector elements enable the robot to achieve motion in a unique direction while retaining the pattern of muscle coordination. In the left of Fig. 3, the muscloskeletal robot seems to move its toe closer to or farther from the hip joint; in particular, it mainly contributes to the moving radius of a toe position, based on the hip joint position. In the right of Fig. 3, the robot seems to perform a rotary motion of the toe position based on the hip joint position, i.e., the argument of the toe position. These results can also be explained with the PC vectors elements. For the first PC vector, the PC element $s_{12}$ corresponding to the A-A ratio $r_{2}$ and the $\mathrm{PC}$ element $s_{13}$ corresponding to the A-A ratio $r_{3}$ respectively indicate opposite signs (Fig. 2). In this case, these elements flex and extend the knee joint synergistically since they have contrary functions for knee joint movement (Table 1). Therefore, muscle coordination represented as the first PC vector of A-A ratio mainly contributes to adjust the distance from the hip joint position to the toe position, relating to knee joint movement. On the other hand, for the sec- ond PC vector, the PC element $s_{22}$ corresponding to the A-A ratio $r_{2}$ and the PC element $s_{23}$ corresponding to the A-A ratio $r_{3}$ have the same sign (Fig. 2). These parameters fix the knee joint movement by counteracting the driving forces around the joint. Furthermore, the PC element $s_{21}$ corresponding to the A-A ratio $r_{1}$ and the PC element $s_{22}$ show the same sign and they have the same function for the hip joint (Table 1), thus they flex and extend the hip joint synergistically. Therefore, muscle coordination represented as the second PC vector of A-A ratio mainly contributes to change the argument of a toe position, based on the hip joint position. The same results were obtained for different subjects at different gait or running speed. This result implies that both human walking and running may be described by a combination of units of motor function that contribute to the argument and the moving radius of a toe position, based on the hip joint position.

\section{Conclusion}

This paper proposed a decomposition of lower limb movements during human walking and running into units of motor function by using PCA for the A-A ratio. The analysis results of human walking and running reveal that (1) all A-A ratios can be decomposed into two patterns of muscle coordination which are represented as $\mathrm{PC}$ vectors, (2) the $\mathrm{PC}$ vectors extracted by A-A ratio analysis do not depend on walking or running speed, or on whether the subject is walking or running, and (3) the two extracted muscle coordination patterns is contribute to the argument and the moving radius of a toe position, based on the hip joint position. Our results suggest that the concept of A-A ratio provide an important clue to solving Bernstein's problem of redundant DFs.

\section{References}

[1] N. Bernstein. The co-ordination and regulation of movements. Oxford, Pergamon, 1967.

[2] G. Cappellini, Y. P. Ivanenko, R. E. Poppele, and F. Lacquaniti. Motor patterns in human walking and running. J. Neurophysiol, 95(6):3426-3437, 2006.

[3] H. Hirai, K. Matsui, T. Iimura, K. Mitsumori, and F. Miyazaki. Modular control of limb kinematics during human walking. Proc. of 2010 IEEE Int. Conf. Biomedical Robotics and Biomechatoronics (BioRob 2010), pages 716-721, 2010.

[4] T. Iimura, K. Inoue, H. T. Pham, H. Hirai, and F. Miyazaki. Decomposition of limb movement based on muscular coordination during human running. $J$. $A d v$. Comp. Intel \& Intel. Informatics, 15(8):980-987, 2011.

[5] http://riodb.ibase.aist.go.jp/dhbodydb/properties/s/index e.html. Human body properties database. 